\title{
COEFFICIENTS OF AN ANALYTIC FUNCTION SUBORDINATION CLASS DETERMINED BY ROTATIONS
}

\author{
SEOK CHAN KIM
}

(Received 8 December 1993; revised 24 June 1994)

Communicated by P. C. Fenton

\begin{abstract}
Let $\mathscr{A}$ denote the set of all functions analytic in $U=\{z:|z|<1\}$ equipped with the topology of uniform convergence on compact subsets of $U$. For $F \in \mathscr{A}$ define

$$
s(F)=\{F \circ \phi: \phi \in \mathscr{A} \text { and }|\phi(z)| \leq|z|\} .
$$

Let $\overline{\operatorname{co}} s(F)$ and $\mathscr{E} \overline{\mathrm{co}} s(F)$ denote the closed convex hull of $s(F)$ and the set of extreme points of $\overline{\mathrm{co}} s(F)$, respectively.

Let $\mathscr{R}$ denote the class of all $F \in \mathscr{A}$ such that $\mathscr{E} \overline{\mathrm{co}} s(F)=\left\{F_{x}:|x|=1\right\}$ where $F_{x}(z)=F(x z)$.

We prove that $\left|A_{N}\right| \leq\left|A_{M N}\right|$ for all positive integers $M$ and $N$, and $(2 \sqrt{2} / 3)\left|A_{2}\right| \leq\left|A_{3}\right|$ for $F(z)=\sum_{N=0}^{\infty} A_{N} z^{N} \in \mathscr{R}$. We also prove that if $F(z)=\sum_{N=0}^{\infty} A_{N} z^{N} \in \mathscr{R}$ and $\left|A_{1}\right|=\left|A_{2}\right|$, then $F$ is a univalent halfplane mapping.
\end{abstract}

1991 Mathematics subject classification (Amer. Math. Soc.): $30 \mathrm{C} 80$.

\section{Introduction}

Let $\mathscr{A}$ denote the set of all functions analytic in $U=\{z:|z|<1\} . \mathscr{A}$ is a linear topological space with respect to the topology of uniform convergence on compact subsets of $U$. Let $F \in \mathscr{A}$ and let $s(F)$ denote the set of all $f \in \mathscr{A}$ such that $f$ is subordinate to $F$. A function $f$ in $\mathscr{A}$ is subordinate to $F$ (written $f \prec F$ ) if there exists $\phi \in \mathscr{B}_{0}=\{\phi \in \mathscr{A}:|\phi(z)| \leq|z|$ for all $z \in U\}$ such that $f=F \circ \phi$. Let $\overline{c o} s(F)$ and $\mathscr{E} \overline{c o} s(F)$ denote the closed convex hull of $s(F)$ and the set of extreme points of $\overline{c o} s(F)$, respectively.

Let $\mathscr{F}$ be a compact subset of $\mathscr{A}$. A function $f \in \mathscr{F}$ is called a support point of $\mathscr{F}$ if there is a continuous linear functional $J$ on $\mathscr{A}$ such that $f$ maximizes $\operatorname{Re} J$ over $\mathscr{F}$

This work was supported by KOSEF 923-0100-009-1

(C) 1996 Australian Mathematical Society $0263-6115 / 95 \$ A 2.00+0.00$ 
and $\operatorname{Re} J$ is nonconstant on $\mathscr{F}$, that is $\operatorname{Re} J(f)=\max \{\operatorname{Re} J(g): g \in \mathscr{F}\}$ and $\operatorname{Re} J$ is nonconstant on $\mathscr{F}$. We use $\Sigma \mathscr{F}$ to denote the set of support points of $\mathscr{F}$.

Let $\mathscr{R}$ denote the class of all $F \in \mathscr{A}$ such that $\overline{c o} s(F)=\left\{\int_{\Gamma} F(x z) d \mu(x): \mu \in \Lambda\right\}$ where $\Lambda$ denotes the set of all probability measures on $\Gamma=\{z:|z|=1\}$. It is worthy of note that $F \in \mathscr{R}$ if and only if $\mathscr{E} \overline{\mathrm{CO}} s(F)=\left\{F_{x}:|x|=1\right\}$ where $F_{x}(z)=F(x z)$. We will show this in Lemma 1.

The problem of finding the general conditions for $F$ to be in $\mathscr{R}$ was posed by T. Sheil-Small. Many examples were shown to be in $\mathscr{R}$ by various authors $([2,3,4$, $6,9,10])$.

The aim of this paper is to find coefficient conditions for $F(z)=\sum_{N=0}^{\infty} A_{N} z^{N}$ to be in $\mathscr{R}$. In [8], D. J. Hallenbeck, S. Perera and D. R. Wilken proved that if $F(z)=\sum_{N=0}^{\infty} A_{N} z^{N} \in \mathscr{R}$ and if $A_{N} \neq 0$, where $N \geq 1$, then $A_{M} \neq 0$ for every $M \geq N$. Here we prove that $\left|A_{N}\right| \leq\left|A_{M N}\right|$ for all positive integers $M$ and $N$, and $2 \sqrt{2} / 3\left|A_{2}\right| \leq\left|A_{3}\right|$ for $F(z)=\sum_{N=0}^{\infty} A_{N} z^{N} \in \mathscr{R}$. We also prove that if $F(z)=\sum_{N=0}^{\infty} A_{N} z^{N} \in \mathscr{R}$ and $\left|A_{1}\right|=\left|A_{2}\right|$, then $F$ is a univalent halfplane mapping.

From the definition of $\mathscr{R}$ we have the following.

FACT 1. $F \in \mathscr{R}$ if and only if $a F+b \in \mathscr{R}$ for all numbers $a, b \in \mathbf{C}$.

FACT 2. $F \in \mathscr{R}$ if and only if $F_{x} \in \mathscr{R},|x|=1$.

So, $F \in \mathscr{R}$ if and only if $e^{i \eta} F\left(e^{i \theta} z\right) \in \mathscr{R}$ for all real $\eta, \theta$.

LEMMA 1. A nonconstant $F \in \mathscr{A}$ is in $\mathscr{R}$ if and only if $\mathscr{E} \overline{C o} s(F)=\left\{F_{x}:|x|=1\right\}$.

PROOF. The sufficiency is obtained by Theorem 1 of [5] and Theorem 5.5 of [7]. Next, we have (with $\mathscr{F}=s(F)$ in $[7$, p.92])

$$
\overline{\mathrm{co}}(\Sigma s(F) \cap \mathscr{E} \overline{\mathrm{co}} s(F))=\overline{\mathrm{co}} s(F) .
$$

To show $F \in \mathscr{R}$, it is enough to show

$$
\Sigma s(F) \cap \mathscr{E} \overline{c o} s(F) \subset\left\{\int_{\Gamma} F(x z) d \mu(x): \mu \in \Lambda\right\} .
$$

If $f \in \Sigma s(F), f=F \circ B$ with $B$ a finite Blaschke product (in [7, p.166]) and $f=F \circ B \in \mathscr{E} \operatorname{co} s(F)=\left\{F_{x}:|x|=1\right\}$ implies $f=F_{x}$ and $f \in\left\{\int_{\Gamma} F(x z) d \mu(x)\right.$ : $\mu \in \Lambda\}$.

LEMMA 2. Let $F \in \mathscr{A}$. If there is a continuous linear functional $J$ and $\varphi \in \mathscr{B}_{0}$ such that $\operatorname{Re} J(F(\varphi))>\operatorname{Re} J\left(F_{x}\right)$ for all $|x|=1$, then $f \notin \mathscr{R}$. 
PROOF. If $F \in \mathscr{R}$, then

$$
\overline{\mathrm{co}} s(F)=\left\{\int_{\Gamma} F(x z) d \mu(x): \mu \in \Lambda\right\} .
$$

So, for any $\varphi \in \mathscr{B}_{0}$, we have

$$
F \circ \varphi \in\left\{\int_{\Gamma} F(x z) d \mu(x): \mu \in \Lambda\right\} .
$$

Thus, for any continuous linear functional $J$ on $\mathscr{A}$, we have

$$
\begin{aligned}
\operatorname{Re} J(F \circ \varphi) & \leq \max _{\mu \in \Lambda} \operatorname{Re} J\left(\int_{\Gamma} F(x z) d \mu(x)\right) \\
& =\max _{\mu \in \Lambda} \int_{\Gamma} \operatorname{Re} J(F(x z)) d \mu(x)=\max _{|x|=1} \operatorname{Re} J\left(F_{x}\right) .
\end{aligned}
$$

\section{Coefficients of elements of the class $\mathscr{R}$}

In this section, we show that, if $F(z)=\sum_{N=0}^{\infty} A_{N} z^{N} \in \mathscr{R},\left|A_{N}\right| \leq\left|A_{M N}\right|$ for every $M, N=1,2,3, \ldots$ and we have that if $|c+1|<1$ then $\exp c((1+z) /(1-z)) \notin \mathscr{R}$ as a corollary. We also show that $(2 \sqrt{2} / 3)\left|A_{2}\right| \leq\left|A_{3}\right|$.

LEMMA 3. If $F(z)=\sum_{N=1}^{\infty} A_{N} z^{N} \in \mathscr{R}$, then

$$
\left|A_{N}\right| \leq\left|A_{M N}\right|, \quad M, N=1,2,3, \ldots
$$

PROOF. Since $F \in \mathscr{R}$, for every $\varphi \in \mathscr{B}_{0}$, there is $\mu \in \Lambda$ such that

$$
F(\varphi(z))=\int_{\Gamma} F(x z) d \mu(x) .
$$

Take $\varphi(z)=z^{M}$. Then

$$
\begin{aligned}
F\left(z^{M}\right) & =\int_{\Gamma} F(x z) d \mu(x) \text { for some } \mu \in \Lambda, \text { that is } \\
\sum_{N=1}^{\infty} A_{N} z^{M N} & =\int_{\Gamma}\left(\sum_{N=1}^{\infty} A_{N} x^{N} z^{N}\right) d \mu(x)=\sum_{N=1}^{\infty} A_{N}\left(\int_{\Gamma} x^{N} d \mu(x)\right) \cdot z^{N} .
\end{aligned}
$$

By considering the coefficient of $z^{M N}$, we have

Hence

$$
\begin{aligned}
A_{N} & =A_{M N} \int_{\Gamma} x^{M N} d \mu(x) . \\
\left|A_{N}\right| & \leq\left|A_{M N}\right| \int_{\Gamma}\left|x^{M N}\right| d \mu(x)=\left|A_{M N}\right| .
\end{aligned}
$$


COROLlaRY 1. If $F=\sum_{N=0}^{\infty} A_{N} z^{N} \in \mathscr{R}$, then $\left|A_{1}\right| \leq\left|A_{M}\right|$ for all $M=$ $1,2,3, \ldots$

ProOF. Let $N=1$ in Lemma 3.

Although the following lemma was proved in [6], we give a shorter proof by using the closedness of $\mathscr{R}$.

LEMMA 4. If Re $c \geq 0$, then $\exp (c(1+z) /(1-z)) \in \mathscr{R}$.

PROOF. Note $(1+w / N)^{N}$ converges uniformly on compact subsets of $U$ to exp $w$ as $N$ goes to $\infty$. Let $\operatorname{Re} c \geq 0$. By a simple calculation we see $\exp (c(1+z) /(1-z))$ is the limit of

$$
f_{N}=\left(\frac{1+((c-N) /(c+N)) z}{1-z}\right)^{N} \cdot\left(\frac{c+N}{N}\right)^{N}, \quad N=1,2,3, \ldots
$$

each of which is in $\mathscr{R}([4])$, since $|(c-N) /(c+N)| \leq 1$. Since $\mathscr{R}$ is closed ([8]), the limit function $\exp (c(1+z) /(1-z))$ is in $\mathscr{R}$.

If $c<0$, then $\exp (c(1+z) /(1-z)) \in H^{1}$ so that $\exp (c(1+z) /(1-z)) \notin \mathscr{R}([1])$. So we conjecture:

$$
\exp \left(c \frac{1+z}{1-z}\right) \notin \mathscr{R} \quad \text { if } \quad \operatorname{Re} c<0 .
$$

Corollary 2 is a partial solution for this.

COROLlARY 2. If $|c+1|<1$, then $\exp (c(1+z) /(1-z)) \notin \mathscr{R}$.

PROOF. Suppose $F(z)=\exp (c(1+z) /(1-z))=\sum_{N=0}^{\infty} A_{N} z^{N} \in \mathscr{R}$. Then we have

$$
A_{1}=2 c \exp c \quad \text { and } \quad A_{2}=\frac{1}{2} \cdot\left(4 c^{2}+4 c\right) \exp c
$$

by simple calculation. By Corollary 1 ,

$$
\left|A_{1}\right| \leq\left|A_{2}\right|, \quad \text { that is } \quad 2|c||\exp c| \leq \frac{1}{2}\left|4 c^{2}+4 c\right||\exp c| \quad \text { or } \quad 1 \leq|c+1| .
$$

This proves the corollary.

To show $(2 \sqrt{2} / 3)\left|A_{2}\right| \leq\left|A_{3}\right|$, we need a technical lemma; 
LEMMA 5. If $r \cos \Phi>\frac{1}{2}, 0<r<1$, then there exists $\theta$ such that

$$
2 \cos \theta-r \cos (\Phi+2 \theta) \geq \sqrt{2} \text {. }
$$

Proof. First, we assume $0 \leq \Phi<\pi / 3$. Let

$$
\begin{aligned}
f(\theta) & =2 \cos \theta-r \cos (\Phi+2 \theta) \\
& =2 \cos \theta-2 r \cos \Phi \cdot \cos ^{2} \theta+r \cos \Phi+2 r \sin \Phi \sin \theta \cos \theta .
\end{aligned}
$$

Let $\theta=\cos ^{-1}(1 / 2 r \cos \Phi)$ with $0<\theta<\pi / 2$. Then $\cos \theta=1 / 2 r \cos \Phi$ and $\sin \theta>0$. Hence

$$
\begin{aligned}
f(\theta) & \geq \frac{1}{r \cos \Phi}-\frac{1}{2 r \cos \Phi}+r \cos \Phi \\
& =\left(\frac{1}{\sqrt{2 r \cos \Phi}}-\sqrt{r \cos \Phi}\right)^{2}+\frac{2}{\sqrt{2}} \geq \frac{2}{\sqrt{2}}=\sqrt{2} .
\end{aligned}
$$

Similarly, we can choose $\theta$ with $-\pi / 2<\theta<0$ for the case $-\pi / 3<\Phi<0$.

REMARK. If $F(z)=A_{1} z+A_{2} z^{2}+A_{3} z^{3}+\cdots$ and $\varphi(z)=b_{1} z+b_{2} z^{2}+b_{3} z^{3}+\cdots$, then

$$
\begin{aligned}
F(\varphi(z))= & A_{1}\left(b_{1} z+b_{2} z^{2}+b_{3} z^{3}+\cdots\right)+A_{2}\left(b_{1} z+b_{2} z^{2}+b_{3} z^{3}+\cdots\right)^{2} \\
& +A_{3}\left(b_{1} z+b_{2} z^{2}+b_{3} z^{3}+\cdots\right)^{3}+\cdots \\
= & A_{1} b_{1} z+\left(A_{1} b_{2}+A_{2} b_{1}^{2}\right) z^{2}+\left(A_{1} b_{3}+2 A_{2} b_{1} b_{2}+A_{3} b_{1}^{3}\right) z^{3}+\cdots
\end{aligned}
$$

THEOREM 1. If $F(z)=\sum_{N=1}^{\infty} A_{N} z^{N}=A_{1} z+A_{2} z^{2}+A_{3} z^{3}+\cdots \in \mathscr{R}$, then

$$
\frac{2 \sqrt{2}}{3}\left|A_{2}\right| \leq\left|A_{3}\right|
$$

ProOF. We may assume $A_{2} \neq 0$ so $A_{3} \neq 0$ ([8]).

By the Facts 1 and 2 in $\S 1, F \in \mathscr{R}$ if and only if $a F(x z) \in \mathscr{R}$ for all $a \in \mathbf{C}$, $|x|=1$. Take

$$
a=\frac{\bar{A}_{2}^{3}}{\left|A_{2}\right|^{4}} \frac{A_{3}^{2}}{\left|A_{3}\right|^{2}} \quad \text { and } \quad x=\frac{A_{2}}{\left|A_{2}\right|} \cdot \frac{\bar{A}_{3}}{\left|A_{3}\right|},
$$

then

$$
\left[z^{2} \text {-coefficient of } a F(x z)\right]=a A_{2} x^{2}=\frac{\bar{A}_{2}^{3}}{\left|A_{2}\right|^{4}} \frac{A_{3}^{2}}{\left|A_{3}\right|^{2}} \cdot A_{2} \cdot \frac{A_{2}^{2}}{\left|A_{2}\right|^{2}} \frac{\bar{A}_{3}^{2}}{\left|A_{3}\right|^{2}}=1
$$


and

$$
\begin{aligned}
{\left[z^{3} \text {-coefficient of } a F(x z)\right] } & =a A_{3} x^{3}=\frac{\bar{A}_{2}^{3}}{\left|A_{2}\right|^{4}} \cdot \frac{A_{3}^{2}}{\left|A_{3}\right|^{2}} \cdot A_{3} \cdot \frac{A_{2}^{3}}{\left|A_{2}\right|^{3}} \cdot \frac{\bar{A}_{3}^{3}}{\left|A_{3}\right|^{3}} \\
& =\frac{1}{\left|A_{2}\right|} \cdot\left|A_{3}\right|>0 .
\end{aligned}
$$

Let $A_{2}=1, A_{3}>0$ and it suffices to show that $A_{3} \geq 2 \sqrt{2} / 3$. Let $A_{1}=r e^{i \Phi}$ and suppose $A_{3}<2 \sqrt{2} / 3$. Then by Corollary 1 we have $r \leq A_{3}<2 \sqrt{2} / 3<1$. We define a continuous linear functional $J$ on $\mathscr{A}$ by $J(f)=a_{3} / A_{3}$ where $f(z)=$ $\sum_{N=0}^{\infty} a_{N} z^{N} \in \mathscr{A}$. Then

$$
\max _{|x|=1} \operatorname{Re} J\left(F_{x}\right)=\max _{|x|=1} \operatorname{Re} \frac{1}{A_{3}} \cdot A_{3} x^{3}=1 .
$$

We will see that there is a $\varphi \in \mathscr{B}_{0}$ such that

$$
\operatorname{Re} J(F \circ \varphi)>1 \quad \text { if } \quad A_{3}<\frac{2 \sqrt{2}}{3}
$$

which will prove $F \notin \mathscr{R}$ by Lemma 2 .

We have two cases

(i) $\operatorname{Re} A_{1} \leq 1 / 2$.

(ii) $\operatorname{Re} A_{1}>1 / 2$, that is $1 / 2<r \cos \Phi$ and $1 / 2<r<A_{3}<2 \sqrt{2} / 3$.

Case (i) Consider

$$
\varphi(z)=\sum_{n=1}^{\infty} b_{n} z^{n}=z \frac{z+\alpha}{1+\bar{\alpha} z}=\alpha z+\left(1-|\alpha|^{2}\right) z^{2}+\bar{\alpha}\left(|\alpha|^{2}-1\right) z^{3}+\cdots
$$

Let $\alpha=1-\varepsilon, 0<\varepsilon<1$, then

$$
b_{1}=1-\varepsilon, \quad b_{2}=2 \varepsilon-\varepsilon^{2}, \quad b_{3}=-2 \varepsilon+3 \varepsilon^{2}-\varepsilon^{3} .
$$

From the remark before the Theorem 1, we have

$$
\begin{aligned}
J(F(\varphi)) & =\frac{1}{A_{3}}\left(A_{1} b_{3}+2 b_{1} b_{2}+A_{3} b_{1}^{3}\right)=b_{1}^{3}+\frac{2}{A_{3}} b_{1} b_{2}+\frac{A_{1}}{A_{3}} b_{3} \\
& =(1-\varepsilon)^{3}+\frac{2}{A_{3}}(1-\varepsilon)\left(2 \varepsilon-\varepsilon^{2}\right)+\frac{A_{1}}{A_{3}}\left(-2 \varepsilon+3 \varepsilon^{2}-\varepsilon^{3}\right)
\end{aligned}
$$

So,

$$
\operatorname{Re} J(F(\varphi))-1=-3 \varepsilon+\frac{2 \varepsilon}{A_{3}}\left[2-\operatorname{Re} A_{1}\right]+\mathscr{O}\left(\varepsilon^{2}\right)
$$


where $\mathscr{O}\left(\varepsilon^{2}\right)$ is such that

$$
\lim _{\varepsilon \rightarrow O^{+}} \frac{\mathscr{O}\left(\varepsilon^{2}\right)}{\varepsilon^{2}}
$$

is finite.

If $\operatorname{Re} A_{1} \leq 1 / 2$, since $A_{3}<1$, there is $\varepsilon>0$ such that

$$
\operatorname{Re} J(F(\varphi))>1=\max _{|x|=1} J\left(F_{x}(z)\right) .
$$

Case (ii) Consider $\varphi_{1}(z)=e^{-i \theta} \varphi\left(e^{i \theta z}\right)$. Let $\alpha=1-\varepsilon, 0<\varepsilon<1$, then

$$
b_{1}=1-\varepsilon, \quad b_{2}=\left(2 \varepsilon-\varepsilon^{2}\right) e^{i \theta}, \quad b_{3}=-\left(2 \varepsilon-3 \varepsilon^{2}+\varepsilon^{3}\right) e^{i \cdot 2 \theta} .
$$

Again from the remark we have

$$
\begin{aligned}
J\left(F\left(\varphi_{1}\right)\right) & =b_{1}^{3}+\frac{2}{A_{3}} b_{1} b_{2}+\frac{A_{1}}{A_{3}} b_{3} \\
& =(1-\varepsilon)^{3}+\frac{2}{A_{3}}\left(2 \varepsilon-3 \varepsilon^{2}+\varepsilon^{3}\right) e^{i \theta}-\frac{A_{1}}{A_{3}}\left(2 \varepsilon-3 \varepsilon^{2}+\varepsilon^{3}\right) e^{i 2 \theta} .
\end{aligned}
$$

So,

$$
\begin{aligned}
\operatorname{Re} J\left(F\left(\varphi_{1}\right)\right) & -1=-3 \varepsilon+\frac{4 \varepsilon}{A_{3}} \operatorname{Re} e^{i \theta}-\frac{2 \varepsilon}{A_{3}} \operatorname{Re} A_{1} e^{i 2 \theta}+\mathscr{O}\left(\varepsilon^{2}\right) \\
= & \varepsilon\left[-3+\frac{2}{A_{3}}(2 \cos \theta-r \cos (\Phi+2 \theta))\right]+\mathscr{O}\left(\varepsilon^{2}\right)
\end{aligned}
$$

By Lemma 5 , there exist $\varepsilon>0$ and $\theta$ such that

$$
\operatorname{Re} J\left(F\left(\varphi_{1}\right)\right)-1>0
$$

which proves $F \notin \mathscr{R}$ in case (ii).

\section{Univalent halfplane mapping}

In this section we prove that if $F(z)=\sum_{N=0}^{\infty} A_{N} z^{N} \in \mathscr{R}$ satisfies $\left|A_{1}\right|=\left|A_{2}\right|$, then $F$ is a univalent halfplane mapping. By the facts in $\S 1$ we may assume $A_{0}=$ 0 and $A_{1}=A_{2}=1$ without loss of generality. We will show $A_{N}=1$ for all $N=3,4,5, \ldots$.

By the definition of $\mathscr{R}$, for every $\varphi \in \mathscr{B}_{0}$, there corresponds a $\mu \in \Lambda$ such that $F(\varphi(z))=\int_{\Gamma} F(x z) d \mu(x)$. For $F(z)=z+z^{2}+\sum_{N=3}^{\infty} A_{N} z^{N} \in \mathscr{R}$, the probability measure $\mu$ which corresponds to $\varphi(z)=z(z+\varepsilon) /(1+\varepsilon z),-1<\varepsilon<1$, is given as in the following lemma. 
LEMMA 6. If $F(z)=z+z^{2}+\sum_{N=3}^{\infty} A_{N} z^{N} \in \mathscr{R}$, the probability measure $\mu$ which corresponds to $\varphi(z)=z(z+\varepsilon) /(1+\varepsilon z),-1<\varepsilon<1$, is

$$
\mu=\left(\frac{1+\varepsilon}{2}\right) \delta_{1}+\left(\frac{1-\varepsilon}{2}\right) \delta_{-1},
$$

where $\delta_{x}$ is point mass at $x$.

ProOF. Let $F(z)=z+z^{2}+\sum_{N=3}^{\infty} A_{N} z^{N} \in \mathscr{R}$. If $\mu$ is the probability measure corresponding to $\varphi(z)=z(z+\varepsilon) /(1+\varepsilon z) \in \mathscr{B}_{0}$, then

$$
\begin{gathered}
F\left(z \frac{z+\varepsilon}{1+\varepsilon z}\right)=\int_{\Gamma}\left(x z+x^{2} z^{2}+\sum_{N=3}^{\infty} A_{N} x^{N} z^{N}\right) d \mu(x) \text { that is } \\
z \frac{z+\varepsilon}{1+\varepsilon z}+\left(z \frac{z+\varepsilon}{1+\varepsilon z}\right)^{2}+\cdots=\int_{\Gamma} x d \mu(x) \cdot z+\int_{\Gamma} x^{2} d \mu(x) \cdot z^{2}+\cdots
\end{gathered}
$$

By comparing the coefficients of the $z-$ ve and $z^{2}-v e$ terms, we have

$$
\int_{\Gamma} x d \mu(x)=\varepsilon \text { and } \int_{\Gamma} x^{2} d \mu(x)=1 .
$$

Let $A=\{1,-1\}, B=\Gamma \backslash A$. Suppose $0<\mu(B) \leq 1$. Then there are a positive number $\eta$ and a subset $B_{0}$ of $B$ such that $0<\mu\left(B_{0}\right)$ and $B_{0}=\{x \in \Gamma:|\operatorname{Im} x| \geq \sin \eta\}$. (Note : $0<\eta<\pi / 2$ ). Then

$$
\begin{aligned}
1 & =\operatorname{Re} \int_{\Gamma} x^{2} d \mu(x)=\operatorname{Re} \int_{B_{0}} x^{2} d \mu(x)+\operatorname{Re} \int_{\Gamma \backslash B_{0}} x^{2} d \mu(x) \\
& \leq\left[\max _{x \in B_{0}} \operatorname{Re} x^{2}\right] \mu\left(B_{0}\right)+\mu\left(\Gamma \backslash B_{0}\right) \leq \sqrt{1-\sin ^{2} \frac{\eta}{2}} \mu\left(B_{0}\right)+\mu\left(\Gamma \backslash B_{0}\right) \\
& <\mu\left(B_{0}\right)+\mu\left(\Gamma \backslash B_{0}\right)=1 .
\end{aligned}
$$

This contradiction gives $\mu(B)=0$ and $\mu(A)=1$. Thus $\mu=\lambda \mu_{1}+(1-\lambda) \mu_{-1}$ with $0 \leq \lambda \leq 1$.

Now, $\int_{\Gamma} x d \mu(x)=\varepsilon$ gives $\lambda=(1+\varepsilon) / 2$, which implies the lemma.

THEOREM 2. If $F(z)=z+z^{2}+\sum_{N=3}^{\infty} A_{N} z^{N} \in \mathscr{R}$, then $A_{N}=1$ for all $N=$ $3,4,5, \ldots$, so that $F$ is a univalent halfplane mapping.

ProOF. By Lemma 6, every $F(z)=z+z^{2}+\sum_{N=3}^{\infty} A_{N} z^{N}$ in $\mathscr{R}$ satisfies

$$
F\left(z \frac{z+\varepsilon}{1+\varepsilon z}\right)=F(z)+\frac{1-\varepsilon}{2}\{F(-z)-F(z)\}
$$


From (*) we have, by differentiating twice with respect to $\varepsilon$,

$$
F^{\prime \prime}\left(z \frac{z+\varepsilon}{1+\varepsilon z}\right) \frac{1-z^{2}}{1+\varepsilon z}-2 F^{\prime}\left(z \frac{z+\varepsilon}{1+\varepsilon z}\right)=0 .
$$

Continuing differentiation, we have

$$
F^{(N+1)}\left(z \frac{z+\varepsilon}{1+\varepsilon z}\right) \frac{1-z^{2}}{1+\varepsilon z}-(N+1) F^{(N)}\left(z \frac{z+\varepsilon}{1+\varepsilon z}\right)=0 .
$$

Let $z=0$. Then we have

$$
F^{(N+1)}(0)-(N+1) F^{(N)}(0)=0
$$

which implies $A_{N}=1$ for all $N=3,4,5, \ldots$

COROLlary 3. If $F(z)=\sum_{N=0}^{\infty} A_{N} z^{N} \in \mathscr{R}$ and $\left|A_{1}\right|=\left|A_{2 N}\right|=1$ for some positive integer $N, F$ is a univalent halfplane mapping.

ProOF. By Lemma 3, we have $\left|A_{1}\right|=\left|A_{2}\right|=1$.

I wish to thank Professor D. R. Wilken, who was my advisor at SUNY at Albany, for providing invaluable assistance at every stage in studing the subject.

\section{References}

[1] Y. Abu-Muhanna, ' $H^{1}$ subordination and extreme points', Proc. Amer. Math. Soc. 95 (1985), 247-251.

[2] - 'Subordination and extreme points', Complex Variables Theory Appl. 9 (1987), 91-100.

[3] Y. Abu-Muhanna and D. J. Hallenbeck, 'A class of analytic functions with integral representations', to appear.

[4] D. A. Brannan, J. G. Clunie and W. E. Kirwan, 'On the coefficient problem for functions of bounded boundary rotation', Ann. Acad. Sci. Fenn. Ser. A. I. Math. 523 (1973).

[5] L. Brickman, T. H. MacGregor and D. R. Wilken, 'Convex hulls of some classical families of univalent functions', Trans. Amer. Math. Soc. 156 (1971), 91-107.

[6] J. Feng, Extreme points and integral means for classes of analytic functions (Ph.D. Thesis, SUNY at Albany, 1974).

[7] D. J. Hallenbeck and T. H. MacGregor, Linear problems and convexity techniques in geometric function theory, Monographs and Studies in Math. 22 (Pitman, Boston, 1984).

[8] D. J. Hallenbeck, S. Perera and D. R. Wilken, 'Subordination, extreme points and support points', Complex Variables Theory Appl. 11 (1989), 111-124.

[9] S. C. Kim, Properties of the family of analytic functions with subordination class determined by rotations (Ph.D. Thesis, SUNY, Albany, 1991). 
[10] S.C. Kim, 'Analytic function with subordination class determined by rotations', Complex Variables Theory Appl. 23 (1993), 177-187.

\section{Department of Mathematics}

Changwon National University

Changwon 641-773

Korea 